\title{
Breast-Specific Gamma Imaging for the Detection of Breast Cancer in Dense Versus Nondense Breasts
}

\author{
Lauren R. Rechtman ${ }^{1}$ \\ Megan J. Lenihan ${ }^{1}$ \\ Jennifer H. Lieberman ${ }^{1}$ \\ Christine B. Teal ${ }^{2}$ \\ Jessica Torrente ${ }^{1}$ \\ Jocelyn A. Rapelyea ${ }^{1}$ \\ Rachel F. Brem ${ }^{1}$
}

Keywords: breast density, breast-specific gamma imaging (BSGI), molecular breast imaging

DOI:10.2214/AJR.13.11585

Received July 15, 2013; accepted after revision September 26, 2013

R. F. Brem is on the board of managers and has stock in Dilon Technologies.

${ }^{1}$ Breast Imaging and Interventional Center, Department of Radiology, The George Washington University, 2150 Pennsylvania Ave NW, Washington, DC 20037. Address correspondence to R. F. Brem (rfbrem@mfa.gwu.edu).

${ }^{2}$ Breast Care Center, Department of Surgery, The George Washington University, Washington, DC.

AJR2014; 202:293-298

0361-803X/14/2022-293

(ㄷ) American Roentgen Ray Society

OBJECTIVE. The purpose of this study was to evaluate the sensitivity of breast-specific gamma imaging (BSGI) for the detection of breast cancer in dense versus nondense breasts.

MATERIALS AND METHODS. This was a retrospective study of 341 women with biopsy-proven breast cancer diagnosed from January 2004 to August 2009 who underwent BSGI before surgical excision. Patients with predominantly fatty replaced (BI-RADS density 1) or scattered fibroglandular tissue (BI-RADS density 2) breasts were classified as nondense, and those with heterogeneously dense (BI-RADS density 3 ) or extremely dense tissue (BIRADS density 4) were classified as dense. BSGI examinations exhibiting focal increased radiotracer uptake in the area of biopsy-proven cancer were classified as positive according to BSGI reports in the medical record. The sensitivity of BSGI was calculated using Microsoft Excel 2003. Between-group differences were evaluated statistically using the Student $t$ test for continuous variables and the chi-square test for categoric variables, with $p<0.05$ considered statistically significant.

RESULTS. The overall sensitivity of BSGI for breast cancer detection was $95.4 \%$. Positive BSGI examinations were present in 136 of 142 nondense breast cancers and 195 of 205 dense breast cancers, for sensitivities of $95.8 \%$ and $95.1 \%$, respectively. There was no significant difference in BSGI breast cancer detection and parenchymal breast density $(p=0.459)$.

CONCLUSION. BSGI has high sensitivities for the detection of breast cancer in women with dense and nondense breasts and is an effective adjunct imaging modality in women with both dense and nondense breasts.

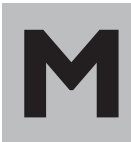
ammography uses an anatomic approach to display physical features of breast cancer within normal surrounding breast tissue [1]. Although mammography remains the standard for breast cancer detection with sensitivity of $85 \%$, the sensitivity decreases to $68 \%$ in women with dense breast tissue [2] and as low as $48 \%$ in women with extremely dense breast tissue [3], making early cancer detection more challenging.

Over half of women younger than 50 years and at least one third of women older than 50 years have either heterogeneously dense or extremely dense breast tissue $[4,5]$. Not only does breast density make mammographic detection of breast cancer more difficult, it is also a strong independent risk factor for developing breast cancer, conferring up to sixfold greater lifetime risk [6-8]. Due to the limitations of mammography in women with dense breasts and an increased risk of developing breast cancer in dense breast tissue, state legislation is increasingly requiring patients to be informed of their breast density. The Breast Density Inform Law, initially enacted in Connecticut in 2009, is now law in 11 states, and bills are endorsed and in progress in numerous other states [9], with the goal of achieving earlier improved cancer detection in this challenging population of women.

Breast-specific gamma imaging (BSGI) is an adjunct modality for breast imaging that, like MRI, uses a physiologic approach to identify lesions in the breast. BSGI has shown sensitivity of $96.4 \%$, comparable with MRI [10], and specificity of $93.3 \%$, greater than MRI [11]. Studies have shown BSGI to be useful in detecting small lesions [10] and occult cancers not found with other imaging modalities [12, 13]. Both MRI and BSGI are physiologic approaches to breast cancer detection, although BSGI has the benefit of being more comfortable, more cost-effective, and less time-consuming for the physician 
to interpret, albeit using a radioactive tracer, which is not used with MRI [14].

Studies of scintimammography using a conventional gamma camera have shown that there is no significant difference in its ability to detect breast cancer in dense versus nondense breast tissue, with sensitivities of $70 \%$ and $72 \%$, respectively [15]. However, the use of scintimammography with a conventional gamma camera for breast imaging is essentially no longer used because of the limited ability to image subcentimeter cancers, image in positions comparable to mammography, and image the entirety of the breast. The development of high-resolution breast-specific gamma cameras, such as in BSGI, has the advantage of a smaller organ-to-detector distance, ability to detect subcentimeter lesions, and imaging in positions comparable with mammography [11]. These factors increase the overall sensitivity of BSGI for breast cancer detection to $96.4 \%$ [10]. To date, there have been no studies investigating the sensitivity of BSGI for the detection of breast cancer in women with dense versus nondense breasts. The purpose of this study was to compare the sensitivity of BSGI in detecting breast cancers in women with dense versus nondense breasts.

\section{Materials and Methods \\ Subjects}

Institutional review board approval and HIPAA full waiver of informed consent were obtained for this study. A retrospective review was performed of all patients who underwent BSGI from January 2004 to August 2009. Three hundred forty-one consecutive patients with newly diagnosed biopsy-proven breast cancer who also underwent BSGI before surgical excision and whose breast density was available from the mammography report were included for analysis. Patients ranged in age from 28 to 89 years (mean age $=55$ years).

\section{BSGI and Interpretation}

BSGI uses a breast-specific, high-resolution, small FOV gamma camera (Dilon 6800, Dilon Technologies). Once in a seated position, patients receive an injection of $15-30 \mathrm{mCi}$ of ${ }^{99 \mathrm{~m}} \mathrm{Tc}$-sestamibi (555-1110 MBq) through an antecubital vein. Imaging begins immediately after injection of the radiotracer, and, because of the nature of the BSGI camera on an articulating arm, the breast can be imaged in all projections, including those positions comparable to mammography. The breast is lightly compressed between two plates and craniocaudal and mediolateral oblique views are obtained at a minimum of 100,000 counts per image. The acquisition time for each image is approximately
6-10 minutes, for a total examination time of 40 45 minutes. The initial images are reviewed by the radiologist and additional views are obtained as deemed clinically necessary. Additional images do not require additional radiotracer injection.

All BSGI examinations were read by radiologists specialized in breast imaging with expertise in BSGI interpretation. BSGI images with increased focal radiotracer uptake in the same breast, quadrant, and distance to the nipple as the biopsy-proven breast cancer were classified as abnormal, and images with no focal radiotracer uptake or scattered heterogeneous physiologic uptake were classified as normal, on the basis of the BSGI report in the patient's medical record. The BSGI examinations were not reinterpreted for this study.

\section{Breast Density Designation}

Mammography was performed in the standard craniocaudal and mediolateral oblique views, with additional images obtained as necessary for clinical care. Breast parenchymal density was determined by the radiology report. Mammograms were interpreted as indicated by the BI-RADS lexicon. The BI-RADS breast density classification was used with 1 , fatty replaced $(<25 \%$ fibroglandular); 2, scattered fibroglandular (25-50\% fibroglandular); 3 , heterogeneously dense $(51-75 \%$ fibroglandular); or 4, extremely dense (> 75\% fibroglandular) breasts [5]. In this study, women with BI-RADS density 1 or 2 were classified as having nondense breasts and those with BI-RADS density 3 or 4 were classified as having dense breasts.

\section{Study Design and Data Collection}

Three hundred forty-one consecutive women with newly diagnosed biopsy-proven breast cancer who underwent BSGI before surgical excision from
January 2004 to August 2009 were included in this study. All women who had a newly diagnosed breast cancer and for whom BSGI was performed for clinical evaluation were included. Women who underwent BSGI after surgical excision were excluded. Family history was noted, including whether there was a first-degree relative with breast cancer (parent, sibling, or child), personal history of breast cancer, age, and menopausal status. Tumor size and pathologic subtype were noted. Positive BSGI examinations were those in which the location of focal radiotracer uptake correlated with the location of biopsy-proven cancer determined with other breast imaging modalities, including mammography, ultrasound, or MRI. BSGI examinations without focal radiotracer uptake in the location of the patient's biopsy-proven cancer were considered negative.

\section{Statistical Analysis}

Data were recorded in a spreadsheet (Microsoft Excel 2003) for analysis. Differences between patients with dense and nondense breasts were analyzed using the Student $t$ test for continuous variables and chi-square test for categoric variables, with $p<0.05$ considered statistically significant. Sensitivity was calculated by the standard method of true-positive findings divided by true-positive findings and false-negative findings.

\section{Results}

\section{Baseline Characteristics}

Patients ranged in age from 28 to 89 years, with a mean age of 55.3 years. One hundred thirteen women $(33.1 \%)$ were premenopausal, 207 women $(60.7 \%)$ were postmenopausal, and 21 women $(6.2 \%)$ were perimenopausal. Ninety of these women $(26.4 \%)$ had a positive family history of breast cancer, defined as hav-

TABLE I: Pathologic Subtype of Biopsy-Proven Breast Cancers

\begin{tabular}{l|c|c}
\multicolumn{1}{c|}{ Type } & No. of Breasts & Percentage \\
\hline Infiltrating ductal carcinoma & 233 & 67.15 \\
Associated DCIS & 145 & 62.23 \\
DCIS & 76 & 21.90 \\
Invasive lobular carcinoma & 26 & 7.49 \\
Associated LCIS & 15 & 57.69 \\
Invasive mammary carcinoma & 3 & $<1$ \\
Papillary carcinoma & 1 & $<1$ \\
Tubular carcinoma & 2 & $<1$ \\
Mucinous adenocarcinoma & 2 & $<1$ \\
Medullary carcinoma & 1 & $<1$ \\
Invasive metaplastic carcinoma & 2 & $<1$ \\
Poorly differentiated carcinoma & 1 & $<1$ \\
\hline
\end{tabular}

Note-DCIS = ductal carcinoma in situ, LCIS = lobular carcinoma in situ. 
ing at least one first-degree relative or multiple second-degree relatives with a history of breast cancer. Thirty-one women $(9.1 \%)$ had personal histories of breast cancer before inclusion in this study. Six women (1.8\%) had bilateral biopsy-proven breast cancer and therefore the analyses were based on 347 breast cancers.

The pathologic findings of the biopsy-proven breast cancers included 233 invasive ductal carcinomas (IDCs) $(67.1 \%), 145$ of which were associated with ductal carcinoma in situ (DCIS), 76 DCIS (21.9\%), 26 invasive lobular carcinomas $(7.5 \%)$, two invasive mammary carcinomas $(<1 \%)$, one papillary carcinoma $(<1 \%)$, two tubular carcinomas $(<1 \%)$, two mucinous adenocarcinomas $(<1 \%)$, one medullary carcinoma $(<1 \%)$, two invasive metaplastic carcinomas $(<1 \%)$, and one poorly differentiated carcinoma $(<1 \%)$ (Table 1$)$. Of the 347 biopsy-proven cancers, nuclear grade was known in 340 cancers and was low grade in 58 cancers $(17.1 \%)$, intermediate grade in 153 cancers $(45.0 \%)$, and high grade in 129 cancers $(37.9 \%)$.

Tumor size was known in 284 of the 347 biopsy-proven breast cancers according to the final pathology report after surgical excision. Cancer size ranged from 0.1 to $12.0 \mathrm{~cm}$, with a mean cancer size of $1.70 \mathrm{~cm}$. Of the 42 cancers with unknown tumor size, pathology reports were not available in the electronic medical records system in 16 cancers. Pathology reports did not specify cancer size in 13 cancers, all of which were DCIS. Administration of neoadjuvant chemotherapy left no residual tumor in seven cancers, three patients chose not to have their tumors excised, and there was no residual tumor left on surgical excision in three cancers.

BSGI examinations were positive in 331 of the 347 biopsy-proven breast cancers, for an overall sensitivity of $95.4 \%$. There were 272 invasive cancers, $263(96.7 \%)$ of which were positive on BSGI, whereas 69 of the 76 DCIS (90.8\%) were positive on BSGI. Of the nine invasive cancers not detected by BSGI, all were IDC making the sensitivity for IDC detection with BSGI $96.1 \%$. BSGI had $100 \%$ sensitivity for the remaining invasive pathologic subtypes of breast cancer, although there were few cancers in each subtype (Table 2). There was no statistically significant difference between BSGI detection and pathologic subtype ( $p=0.428)$. Fifteen of the breast cancers with negative BSGI examinations were detected on mammography, whereas one breast cancer was only detected on ultrasound.

Nuclear grade was known in 325 cancers detected by BSGI and was low grade in 55
TABLE 2: Sensitivity of Breast-Specific Gamma Imaging (BSGI) on Basis of Pathologic Subtype of Breast Cancer

\begin{tabular}{l|c|c}
\hline \multicolumn{1}{c|}{ Type $^{\text {a }}$} & Positive BSGI Examinations/Total & Sensitivity (\%) \\
\hline Infiltrating ductal carcinoma & $224 / 233$ & 96.1 \\
Ductal carcinoma in situ & $69 / 76$ & 90.8 \\
Invasive lobular carcinoma & $26 / 26$ & 100 \\
Other $^{b}$ & $12 / 12$ & 100
\end{tabular}

aBetween-group differences were calculated using the chi-square test $(p=0.428)$.

${ }^{b}$ Other is defined as papillary carcinoma, tubular carcinoma, mucinous adenocarcinoma, medullary carcinoma, invasive metaplastic carcinoma, and poorly differentiated carcinoma.

TABLE 3: Characteristics of Study Participants According to Breast Density

\begin{tabular}{l|c|c}
\hline \multicolumn{1}{c|}{ Characteristic } & Nondense Breasts & Dense Breasts \\
\hline Age $^{\mathrm{a}}(\mathrm{y})$ & & $88 / 205(42.9)$ \\
$<50$ & $41 / 142(28.9)$ & $75 / 205(36.6)$ \\
$50-64$ & $58 / 142(40.8)$ & $42 / 205(20.5)$ \\
$\geq 65$ & $43 / 142(30.3)$ & 53.4 \\
Mean $^{\mathrm{b}}$ & 58.1 & \\
Race $^{\mathrm{a}}$ & & $104 / 205(50.7)$ \\
White $^{\prime}$ & $47 / 142(33.1)$ & $79 / 205(38.5)$ \\
African American & $82 / 142(57.7)$ & $13 / 205(6.3)$ \\
Asian & $7 / 142(4.9)$ & $7 / 205(3.4)$ \\
Hispanic & $4 / 142(2.8)$ & $2 / 205(<1)$ \\
Unknown & $2 / 142(1.4)$ & $80 / 205(39.0)$ \\
Menopausal status & & $16 / 205(7.8)$ \\
Premenopausal & $34 / 142(23.9)$ & $109 / 205(53.2)$ \\
Perimenopausal & $5 / 142(3.5)$ & \\
Postmenopausal & $103 / 142(72.5)$ & \\
\hline
\end{tabular}

Note-Data are number/total with percentage in parentheses.

a Statistically significant between-group differences were calculated using the chi-square test $(p \leq 0.017)$.

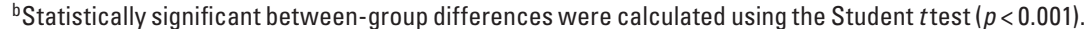

TABLE 4: Detection of Pathologic Subtypes and Nuclear Grades According to Breast Density (Sensitivity)

\begin{tabular}{l|c|c|c}
\hline \multicolumn{1}{c|}{ Characteristic } & Nondense Breasts & Dense Breasts & $p^{\text {a }}$ \\
\hline Pathologic subtype & & & 0.087 \\
Infiltrating ductal carcinoma & $97 / 100(97.0)$ & $127 / 133(95.5)$ & \\
Ductal carcinoma in situ & $23 / 26(88.5)$ & $46 / 50(92.0)$ & \\
Invasive lobular carcinoma & $8 / 8(100)$ & $18 / 18(100)$ & \\
Other & $8 / 8(100)$ & $4 / 4(100)$ & 0.509 \\
Nuclear grade & & & \\
Low & $26 / 29(89.7)$ & $29 / 29(100)$ & \\
Intermediate & $60 / 62(96.8)$ & $84 / 91(92.3)$ & \\
High & $48 / 49(98.0)$ & $78 / 80(97.5)$ & \\
\hline
\end{tabular}

Note-Data are number/total with percentage in parentheses.

abetween-group differences were calculated using the chi-square test.

${ }^{\mathrm{b}}$ Other is defined as papillary carcinoma, tubular carcinoma, mucinous adenocarcinoma, medullary carcinoma, invasive metaplastic carcinoma, and poorly differentiated carcinoma. 
cancers $(16.9 \%)$, intermediate grade in 144 cancers $(44.3 \%)$, and high grade in 126 cancers $(38.8 \%)$. Nuclear grade was known in 15 cancers undetected by BSGI: nine invasive and six DCIS. Of all the cancers not detected with BSGI, three $(18.7 \%)$ were low grade, nine $(56.2 \%)$ were intermediate grade, and three $(18.7 \%)$ were high grade, with no significant difference between BSGI detection and nuclear grade ( $p=0.256)$. Of the nine invasive cancers undetected by BSGI, six were intermediate grade and two were low grade. BSGI detected all high-grade, invasive cancers.

Tumor size was available in 272 of 331 $(82.2 \%)$ positive BSGI examinations (mean, $1.74 \mathrm{~cm})$ and 12 of $16(75.0 \%)$ negative BSGI examinations (mean, $1.02 \mathrm{~cm}$ ) as tumorsize for DCIS is often not reported. The lower percentage of cancers with known size and negative BSGI reflects the lower sensitivity of BSGI for DCIS because a greater percentage of cancers with unknown size were DCIS. There was no statistically significant difference in tumor size for cancers detected with BSGI and those that were not ( $p=0.065)$. Of the 347 breast cancers, 162 were palpable on physical examination $(46.7 \%)$. One hundred fifty-eight of these were detected on BSGI $(97.5 \%)$ and four were not $(2.5 \%)$.

\section{Dense Versus Nondense Breasts}

The BI-RADS mammography parenchymal density in the 347 breasts were designated BI-RADS density 1 in 47 (13.5\%), 2 in 94 (27.1\%), 3 in $166(47.8 \%)$, and 4 in $40(11.5 \%)$. On the basis of the classification for this study, 141 breasts were nondense $(40.6 \%)$ and 206 breasts were dense $(59.4 \%)$. The mean age of women with dense breasts was 53.4 years and the mean age of women with nondense breasts was 58.1 years, again showing that women with dense breasts are significantly younger when compared with women with nondense breasts $(p=0.0004)$. Similarly, significantly more women with dense breasts were pre-

\section{Rechtman et al.}

TABLE 5: Lesion Size and Breast-Specific Gamma Imaging Sensitivity According to Breast Density

\begin{tabular}{l|c|c|c}
\hline \multicolumn{1}{c|}{ Characteristic } & Nondense breasts & Dense breasts & $p^{\mathrm{a}}$ \\
\hline Lesion size $(\mathrm{cm})$ & & & 0.622 \\
$0.1-0.49$ & $11 / 11(100)$ & $12 / 15(80.0)$ & \\
$0.5-0.99$ & $22 / 23(95.7)$ & $25 / 27(92.6)$ & \\
$1.0-1.99$ & $58 / 61(95.1)$ & $60 / 62(96.8)$ & \\
$2.00-4.99$ & $30 / 30(100)$ & $48 / 49(98.0)$ & \\
$>5.00$ & $2 / 2(100)$ & $4 / 4(100)$ & \\
Unknown & $12 / 13(92.3)$ & $41 / 43(95.3)$ & \\
\hline
\end{tabular}

Note-Data are number/total with percentage in parentheses.

${ }^{a} B$ Between-group differences were calculated using the chi-square test.

menopausal, whereas fewer were postmenopausal compared with women with nondense breasts $(p=0.001)$ (Table 3).

The pathologic subtype of breast cancers consisted of IDC in 100 nondense $(70.4 \%)$ and 133 dense $(64.9 \%)$ breasts, DCIS in 26 nondense $(18.3 \%)$ and 50 dense $(24.4 \%)$ breasts, infiltrating lobular carcinoma in eight nondense $(5.6 \%)$ and 18 dense $(8.8 \%)$ breasts, and various other pathologic subtypes in eight nondense $(5.6 \%)$ and four dense $(1.9 \%)$ breasts. Of the 347 cancers, 340 had known nuclear grade. The cancers included 29 lowgrade cancers in women with dense breasts (14.5\%) and 29 low-grade cancers in women with nondense breasts $(20.7 \%)$; 91 intermediate-grade cancers were found in women with dense breasts (45.5\%), and 63 intermediate-grade cancers in women with nondense breasts (44.3\%); 80 high-grade cancers were seen in women with dense breasts $(40.0 \%)$ and 49 were seen in women with nondense breasts $(35.0 \%)$. There was no statistically significant difference between breast density and pathologic subtype $(p=0.102)$ or nuclear grade ( $p=0.296$ ) (Table 4$)$. Of the 284 cancers in which tumor size was available, there was no statistically significant difference between breast density and breast cancer size in BSGI sensitivity ( $p=0.622)$, with a mean tu- mor size of $1.64 \mathrm{~cm}$ in nondense breasts and $1.76 \mathrm{~cm}$ in dense breasts (Table 5).

Cancers in 136 of the 141 women with nondense breasts and in 194 of the 206 women with dense breasts were positive for sensitivities of $96.5 \%$ and $94.7 \%$, respectively (Table 6). Of the 16 breast cancers not detected by BSGI, five $(31.3 \%)$ were in nondense and 11 $(68.8 \%)$ were in women with dense breasts. There was no statistically significant difference between breast parenchymal density and BSGI breast cancer detection $(p=0.459)$.

Twenty of the 347 breast cancers (5.8\%) were mammographically occult, five of which were in nondense breasts and 15 of which were in dense breasts. Of these, BSGI detected all five cancers in women with nondense breasts (100\% sensitivity) and 14 of 15 in women with dense breasts $(93.3 \%$ sensitivity). The one breast cancer that was occult on both mammography and BSGI was palpable and visible on ultrasound. There was no statistically different sensitivity for BSGI detection of mammographically occult breast cancer in women with dense and nondense breasts $(p=0.102)($ Fig. 1$)$.

\section{Discussion}

The purpose of this study was to evaluate BSGI in women with dense versus nondense

TABLE 6: Sensitivity of Breast-Specific Gamma Imaging (BSGI) According to Breast Density

\begin{tabular}{l|c|c|c}
\hline \multicolumn{1}{c|}{ Breast Density $^{\text {a }}$} & Positive BSGI Examinations & Negative BSGI Examinations $^{\text {Sensitivity (\%) }}$ \\
\hline Nondense breasts & $136 / 141$ & $5 / 141$ & 96.5 \\
BI-RADS 1 & $44 / 47$ & $3 / 47$ & 93.6 \\
BI-RADS 2 & $92 / 94$ & $2 / 94$ & 97.9 \\
Dense breasts & $195 / 206$ & $11 / 206$ & 94.7 \\
BI-RADS 3 & $160 / 166$ & $8 / 166$ & 95.2 \\
BI-RADS 4 & $35 / 40$ & $3 / 40$ & 92.1 \\
\hline
\end{tabular}

Note-Data are number/total.

aBetween-group differences were calculated using the chi-square test $(p=0.459)$. 
breast tissue. This study shows that the sensitivity of BSGI was similar in women with dense $(94.7 \%)$ and nondense breasts $(96.5 \%)$, with no significant difference between BSGI cancer detection and breast density ( $p=$ 0.459). Consistent with previous studies showing that scintimammography is beneficial in women of all breast densities [7, 15], this study shows that BSGI is also a useful adjunct imaging modality in the diagnosis of breast cancer in women with both dense and nondense breasts. Our study also showed that there were more mammographically occult breast cancers in women with dense breast tissue, although, BSGI detected mammographically occult breast cancers equally well in women with dense and nondense breast tissue. This study corroborates others that have shown that BSGI can detect mammographically occult breast cancer, although our study now shows that BSGI can detect mammographically occult breast cancer equally well in women with dense and nondense breasts.

Improving breast cancer detection in women with dense breasts is important because of the decreased sensitivity of mammography in this population [2]. Although the combination of ultrasound and mammography in women with dense breasts and other highrisk populations increases the sensitivity, it also substantially increases the false-positive rate [16-18]. MRI, which uses a physiologic approach to breast cancer detection, has been established as a useful adjunct breast imaging modality because of its increased sensitivity compared with ultrasound and mammography and its effectiveness in women of all breast densities [19-22]. The availability of BSGI now offers those women who cannot or will not undergo MRI an alternative for physiologic imaging. The addition of BSGI expands our armamentarium of imaging modalities for improved detection of breast cancer. Previous studies have shown that BSGI has high sensitivity and specificity for the detection of breast cancer. This study shows that the sensitivity of BSGI is not significantly different in women with dense breasts compared with those with nondense breasts.

The overall sensitivity of BSGI was $95.4 \%$, with sensitivities of $96.1 \%$ for IDC, $90.8 \%$ for DCIS, and $100.0 \%$ for invasive lobular carcinoma. These results are comparable to previously published BSGI sensitivities ranging from $91.0 \%$ to $97.6 \%$ [14, 22, 23]. This study also shows that the ability of BSGI to detect breast cancer is not affected by pathologic subtype $(p=0.102)$, nuclear grade $(p=0.296)$, or

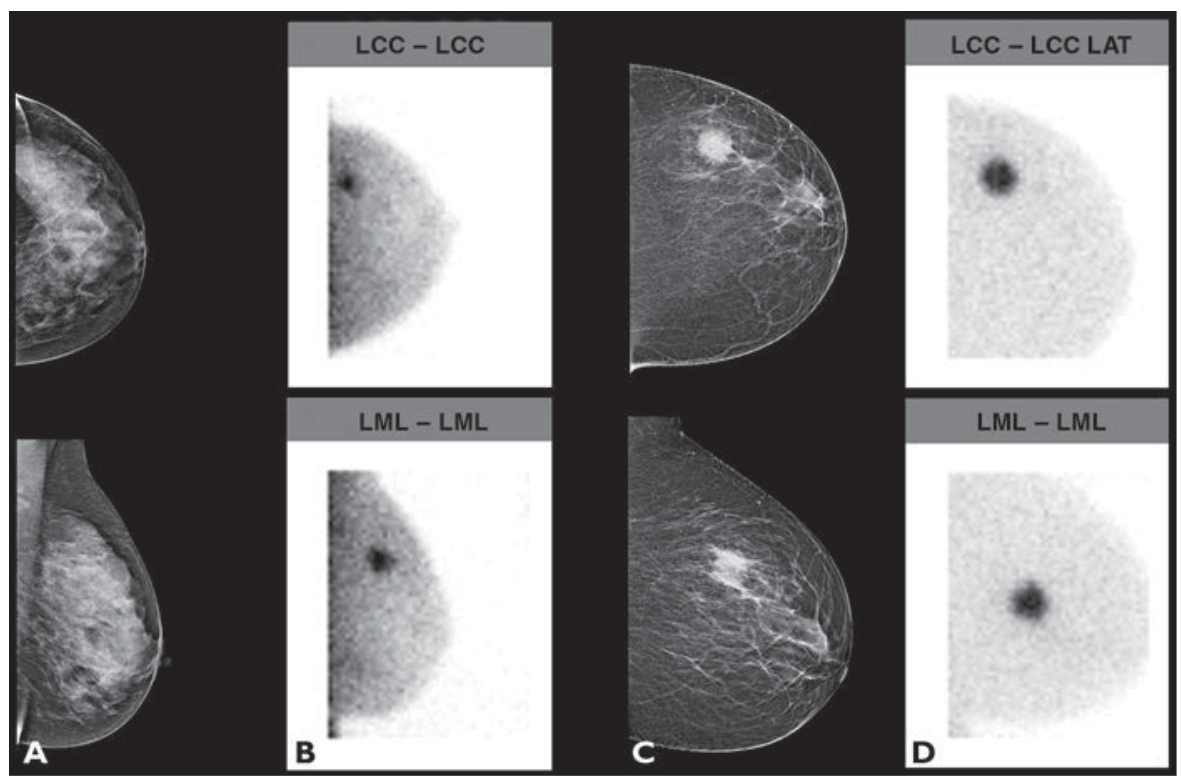

Fig. 1-Comparison of dense and nondense breast imaging with mammography and BSGI.

$\mathbf{A}$ and $\mathbf{B}$, Images from mammography (A) and breast-specific gamma imaging (BSGI) (B) in 48-year-old woman with heterogeneously dense breast tissue show infiltrating ductal carcinoma (IDC) in superior left breast. C and D, Images from mammography (C) and breast-specific gamma imaging (BSGI) (D) in 53-year-old woman with predominantly fatty replaced breast tissue show IDC in 2-o'clock position of left breast.

tumor size $(p=0.065)$. However, it is of note that none of the invasive breast cancers with a high nuclear grade were missed with BSGI. Although this study did not show a difference in BSGI detection of breast cancer on the basis of nuclear grade, that finding is likely because both invasive and noninvasive breast cancers were grouped together. A previous study which showed that nuclear grade is a factor in BSGI detection of cancer included only invasive breast cancers. Our study did show that all high-grade invasive cancers were detected with BSGI, which corroborated a previous report that similarly showed that all high-grade invasive cancers were detected with BSGI [24]. With regard to invasive lobular carcinoma, our study corroborates previous reports that did not show a decrease in sensitivity for detection of invasive lobular carcinoma using BSGI compared with mammography $[14,25]$, ultrasound $[14,26]$, and MRI $[14,26]$.

In this population of women, the false-negative rate of BSGI was $4.6 \%$ (16/347). BSGI detected 19 of 20 (95\%) mammographically occult breast cancers, which is consistent with previous findings that BSGI is effective in the identification of occult breast cancers [13]. Of the 19 mammographically occult cancers detected using BSGI, 14 (73.6\%) were in women with dense breasts. These results suggest that BSGI can be especially beneficial to women with dense breasts because of its ability to identify breast cancer missed at mammography and its high sensitivity in this population.

Although both MRI and BSGI use a physiologic approach to breast cancer detection and have been shown to be useful adjunct imaging modalities, BSGI uses a low-dose radiotracer and thus necessitates radiation exposure, whereas MRI is performed without the use of ionizing radiation. However, in the correct population, this is a reasonable tradeoff for early breast cancer detection. In this study, a dose of 20-30 mCi ${ }^{99 m}$ TC sestamibi was used. We now routinely use a lower dose of 7-10 $\mathrm{mCi}$.

This study shows that BSGI is equally sensitive for the detection of breast cancer in women with dense as well as nondense breast tissue. Although this study did not directly compare BSGI with MRI, previous studies have reported comparable sensitivity and higher specificity for BSGI compared with MRI [7, 10, 20]. BSGI has additional advantages over MRI, including lower costs, less time for interpretation, and more women who can undergo the examination. To our knowledge, there have been no studies specifically comparing sensitivity of MRI in women with dense versus nondense breasts, and additional studies are needed to compare the sensitivity of BSGI and MRI in such women.

Limitations of this study include its retrospective nature and single institutional review. Additionally, the inclusion criteria of 


\section{Rechtman et al.}

this study limited the population of women to those with newly diagnosed breast cancer, which can introduce bias. Further studies should compare the sensitivity and specificity of BSGI in women with dense and nondense breasts in a multiinstitutional population of women undergoing screening.

In conclusion, the results of this study have shown that BSGI is an effective imaging modality in women of all breast densities, with equal sensitivities in women with dense and nondense breasts. Its high accuracy and effectiveness regardless of breast density specifically support the use of BSGI in the clinical evaluation of women with dense breasts, with the goal of achieving earlier cancer detection in this population of women.

\section{Acknowledgments}

We thank Anita P. McSwain and Caitrin M. Coffey of The George Washington University for their contributions to this study.

\section{References}

1. Brem RF, Rechtman LR. Nuclear medicine imaging of the breast: a novel, physiologic approach to breast cancer detection and diagnosis. Radiol Clin North Am 2010; 48:1055-1074

2. Rosenberg RD, Hunt WC, Williamson MR, et al. Effects of age, breast density, ethnicity, and estrogen replacement therapy on screening mammographic sensitivity and cancer stage at diagnosis: review of 183,134 screening mammograms in Albuquerque, New Mexico. Radiology 1998; 209:511-518

3. Kolb TM, Lichy J, Newhouse JH. Comparison of the performance of screening mammography, physical examination, and breast US and evaluation of factors that influence them: an analysis of 27,825 patient evaluations. Radiology 2002; 225:165-175

4. Stomper PC, D'Souza DJ, DiNitto PA, Arredondo MA. Analysis of parenchymal density on mammograms in 1353 women $25-79$ years old. AJR 1996; 167:1261-1265

5. D’Orsi CJ, Mendelson EB, Ikeda EM, et al. BI-
RADS: mammography, 4th ed. In: D'Orsi CJ, Mendelson EB, Ikeda EM, et al. Breast Imaging Reporting and Data System: ACR. Reston, VA: American College of Radiology, 2003

6. Harvey JA, Bovbjerg VE. Quantitative assessment of mammographic breast density: relationship with breast cancer risk. Radiology 2004; 230:29-41

7. Brem RF, Rapelyea JA, Zisman G, et al. Occult breast cancer: scintimammography with highresolution breast-specific gamma camera in women at high risk for breast cancer. Radiology 2005; 237:274-280

8. Boyd NF, Guo H, Martin LJ, et al. Mammographic breast density and the risk and detection of breast cancer. N Engl J Med 2007; 356:227-236

9. Are You DENSE Advocacy website. www.areyoudenseadvocacy.org/dense. Accessed October 3, 2013

10. Brem RF, Floerke AC, Rapelyea JA, Teal C, Kelly T, Mathur V. Breast-specific gamma imaging as an adjunct imaging modality for the diagnosis of breast cancer. Radiology 2008; 247:651-657

11. Brem RF, Schoonjans JM, Kieper DA, et al. Highresolution scintimammography: a pilot study. $J$ Nucl Med 2002; 43:909-915

12. Zhou M, Johnson N, Gruner S, et al. Clinical utility of breast-specific gamma imaging for evaluating disease extent in the newly diagnosed breast cancer patient. Am J Surg 2009; 197:159-163

13. Brem RF, Shahan C, Rapelyea JA, et al. Detection of occult foci of breast cancer using breast-specific gamma imaging in women with one mammographic or clinically suspicious breast lesion. Acad Radiol 2010; 17:735-743

14. Brem RF, Ioffe M, Rapelyea JA, et al. Invasive lobular carcinoma: detection with mammography, sonography, MRI, and breast-specific gamma imaging. AJR 2009; 192:379-383

15. Khalkhali I, Baum JK, Villanueva-Meyer J, et al. ${ }^{99 \mathrm{~m}} \mathrm{Tc}$ sestamibi breast imaging for the examination of patients with dense and fatty breasts: multicenter study. Radiology 2001; 222:149-155

16. Kaplan SS. Clinical utility of bilateral wholebreast US in the evaluation of women with dense breast tissue. Radiology 2001; 221:641-649

17. Leconte I, Feger C, Galant C, et al. Mammography and subsequent whole-breast sonography of nonpalpable breast cancers: the importance of radiologic breast density. AJR 2003; 180:1675-1679

18. Berg WA, Blume JD, Cormack JB, et al. Combined screening with ultrasound and mammography vs mammography alone in women at elevated risk of breast cancer. JAMA 2008; 299:2151-2163

19. Irwig L, Houssami N, van Vliet C. New technologies in screening for breast cancer: a systematic review of their accuracy. Br J Cancer 2004; 90:2118-2122

20. Brem RF, Petrovitch I, Rapelyea JA, Young H, Teal C, Kelly T. Breast-specific gamma imaging with ${ }^{99 \mathrm{~m}}$ Tc-sestamibi and magnetic resonance imaging in the diagnosis of breast cancer: a comparative study. Breast J 2007; 13:465-469

21. Weigert JM, Bertrand ML, Lanzkowsky L, Stern LH, Kieper DA. Results of a multicenter patient registry to determine the clinical impact of breastspecific gamma imaging, a molecular breast imaging technique. AJR 2012; 198:[web]W69-W75

22. Elmore JG, Armstrong K, Lehman CD, Fletcher SW. Screening for breast cancer. JAMA 2005; 293:1245-1256

23. Brem RF, Fishman M, Rapelyea JA. Detection of ductal carcinoma in situ with mammography, breast specific gamma imaging, and magnetic resonance imaging: a comparative study. Acad Radiol 2007; 14:945-950

24. Tadwalkar RV, Rapelyea JA, Torrente J, et al. Breast-specific gamma imaging as an adjunct modality for the diagnosis of invasive breast cancer with correlation to tumour size and grade. $\mathrm{Br} J$ Radiol 2012; 85:e212-e216

25 . Krecke KN, Gisvold JJ. Invasive lobular carcinoma of the breast: mammographic findings and extent of disease at diagnosis in 184 patients. AJR 1993; 161:957-960

26. McGhan LJ, Wasif N, Gray RJ, et al. Use of preoperative magnetic resonance imaging for invasive lobular cancer: good, better, but maybe not the best? Ann Surg Oncol 2010; 17:255-262 